\title{
Editorial
}

Nephrology

Published online: January 14, 2020

\section{Circulating Endocannabinoid Levels as Potential Biomarkers for Mortality in Hemodialysis Patients}

\author{
Ilia Beberashvili
}

Division of Nephrology, Shamir Medical Center (Assaf Harofeh), Affiliated with Sackler Faculty of Medicine Tel Aviv University, Zerifin, Israel

Many biomarkers related to uremia-attributed nontraditional risk factors, such as mineral and bone disorders, anemia, protein-energy wasting, inflammation and oxidative stress, have been recently tested in the end-stage kidney disease (ESKD) population and were found to be associated with decreased survival [1]. While not all of them are in clinical use, researching mortality biomarkers is still of paramount importance. Along with providing a dynamic approach to our understanding of the etiology and pathophysiology of organ system alterations, they may allow us to define high-risk patient groups and identify surrogate endpoints for important clinical outcomes [2]. From this perspective, the recently discovered endocannabinoid (EC) system requires special attention, especially due to the therapeutic potential of cannabis in such diseases as obesity and metabolic syndrome, diabetes, neurodegenerative, inflammatory, cardiovascular, and gastrointestinal diseases among many others [3].

Despite records going back 4,700 years that document medicinal uses of cannabis, its mechanism of action was unknown until 1964 when Yechiel Gaoni and Raphael Mechoulam reported on the main active component of cannabis, $\Delta 9$-tetrahydrocannabinol ( $\Delta 9$-THC) [4]. The first reported receptor sites, named cannabinoid (CB) receptors, in mammalian brains that respond to $\triangle 9$-THC

\section{KARGER}

(c) 2019 S. Karger AG, Basel

E-Mail karger@karger.com

www.karger.com/ajn were reported in 1988, and the DNA sequence of one such brain CB receptor was mapped in 1990 [5]. Two CB receptors, $\mathrm{CB} 1$ and later $\mathrm{CB} 2$, were also isolated and cloned that year [5].

The EC system encompasses 2 types of G-protein-coupled receptors, $\mathrm{CB} 1$ and $\mathrm{CB} 2$, endogenous ligands of these receptors, known as "ECs," anandamide (arachidonoyl ethanolamide [AEA]) and 2-arachidonoyl glycerol (2-AG), and the processes responsible for EC biosynthesis, cellular uptake, and degradative metabolism [5]. Structurally, both AEA and 2-AG are arachidonic acidcontaining lipid molecules. However, 2-AG is a full agonist of the central receptor CB1 as well as the peripheral receptor $\mathrm{CB} 2$, whereas anandamide is a partial agonist of both receptors. CB1 receptors are the most abundant receptors in the mammalian brain but are also present at much lower concentrations in peripheral organs such as the gastrointestinal tract $[3,5]$. CB1 activation contributes to the $\mathrm{CB}$ effects on cognitive impairment and depressed motor function, influences gut motility, promotes energy storage, and impairs glucose and lipid metabolism. CB2 receptors are expressed primarily in cells of the immune and hematopoietic systems resulting in immunosuppressive and anti-inflammatory responses via inhibition of neutrophil migration, suppression of 
proinflammatory factor proliferation, and a reduction of signaling to T cells. Recently, CB2 receptors were also found in the brain, in nonparenchymal cells of the cirrhotic liver, in the endocrine pancreas, and in bone [5] ECs therefore oversee complex crosstalk between the central and peripheral nervous system and the gut, muscle, and adipose tissue $[3,5,6]$. ECs may therefore exert a complex of desirable (pain relief, reduction of nausea/ vomiting, insulin resistance, inflammation, lipogenesis, depression, and stimulation of appetite, lipolysis, glucose tolerance) and undesirable (decrease in myocardial contractility and fertility and an increase in psychoactive effects and gastrointestinal motility) effects [3, 5]. ECs therefore may represent biomarkers of clinical outcomes including mortality in high-risk populations such as maintenance hemodialysis (MHD) population. However, so far, there are no data in the existing literature linking serum EC levels and survival. This explains the special interest in the paper by Moradi et al. [7] published in the current issue of the American Journal of Nephrology.

To study the association of circulating ECs with mortality in MHD patients, Moradi et al. [7] analyzed 436 hemodialysis patients from 523 participants of the malnutrition, diet, and racial disparities in chronic kidney disease study with available serum EC measurements performed between October 2013 and 2015. These patients were followed for 12 months. In a multivariate analyses adjusted for a number of risk factors including demographic and clinical parameters (age, sex, race, ethnicity, diabetes and dialysis vintage) and inflammation biomarkers like IL-6, investigators found that the study patients with higher serum $2-\mathrm{AG}(\geq 32.5 \mathrm{pmol} / \mathrm{mL})$ had lower 12-month all-cause mortality risks compared to the group with lower serum $2-\mathrm{AG}(<32.5 \mathrm{pmol} / \mathrm{mL})$ with an HR (95\% CI) of $0.52(0.28-0.98) \mathrm{pmol} / \mathrm{mL}$. Conversely, the study found that AEA did not predict 12-month allcause mortality risk in MHD patients. This discriminatory association between 2 ECs and mortality risk lead to the assumption of their different in vivo roles. However, the specific biological significance of AEA and 2-AG as agonists of CB1 and CB2 has not been fully elucidated [3, 5]. Additionally, oppositely directed ECs levels in MHD patients versus the general population and a relatively short follow-up time could also have influenced the results of the study. The same authors of an article published in this issue of the Journal that we comment on [7] have recently reported on increased 2-AG and decreased AEA concentrations in MHD patients, measured in 2 different laboratories and 2 separate sets of patients with age-, sex-, race-, and ethnicity-matched controls [8]. Al- though 2-AG demonstrates characteristics of a mortality biomarker in MHD patients in the study by Moradi et al. [7], the question is whether 2-AG has real biological interference with the pathophysiological mechanism(s) that may influence clinical outcomes in ESKD patients. It is well known that increased levels of certain solutes that may signal a high death risk in ESKD patients reflect mostly the severity of the uremic syndrome or alterations triggered by ESKD rather than the true associations of these solutes with mortality risk. Such a confounding is very characteristic for observational studies in MHD population [2]. However, in this study, 2-AG is inversely rather than directly associated with death risk, excluding the confounding by accumulation attributable to ESKD in the interpretation of the results. The next question that immediately arises here is to what extent the link between 2-AG and mortality risk is confounded by traditional Framingham risk factors and nontraditional uremia-related risk factors in the study population. In this respect, appropriately adjusted analyses might help in understanding the reliable underlying biological role of 2-AG in predicting the study population's mortality risk. However, multivariate analyses presented by the authors [7] are lacking several confounders that may influence survival in the MHD population (e.g., cardiovascular comorbidities, residual renal function, dietary intake, nutritional status evaluation, oxidative stress markers), so the above question remains only partially answered. This is the study's major limitation.

The possible link between circulating 2-AG and mortality in MHD patients may be explained by several mechanisms. First, EC signaling is a potent mediator of food intake and influences energy metabolism through its regulation of orexigenic and anorectic molecules and neural pathways [3]. The therapeutic effectiveness of synthetic $\Delta 9$-THC (the main psychoactive constituent of cannabis), or even smoked marijuana as appetite boosters or stimulator of weight gain, has been described in patients with cancer and AIDS [3,5]. However, whether uremia contributes to anorexia via the EC system is unknown and requires further research. Second, 2-AG-mediated prevention of brown adipose tissue activation (that is involved mainly in thermogenesis and caloric expenditure during resting and exercises [5]), as well as of "browning" of white adipose tissue, may lead to energy conservation and can be the possible link between EC system activity and mortality in MHD patients. A positive energy balance resulting from decreased energy expenditure, leading to prevention of weight loss, can consequently prevent protein energy wasting and thus can affect survival of ESKD 
patients receiving MHD [1]. The positive association between 2-AG and body mass index and especially with fat mass surrogate measures (triceps skinfold and body fat percent measured by near-infrared interactance), presented by Moradi et al. [7], can support this assumption. Third, the selective activation of CB2 appears to exert cardioprotective effects by protecting against oxidative stress-induced tissue damage in experimental models of ischemia-reperfusion injury, inflammation, and/or atherosclerosis [6]. Most of the aforementioned pathways through which ECs are linked to mortality in HD patients are, however, the subjects of future preclinical and clinical studies.
Overall, in this intriguing and hypothesis-generating cohort study, Moredi et al. [7] apparently support the role of ECs, particularly of 2-AG, as potentially relevant players in ESKD's high risk of death. Given the study's interesting findings, focusing on ECs seems to be a worthwhile research effort for investigators in the field, especially in targeting ECs for therapeutic gain in the ESKD population.

\section{Disclosure Statement}

The author declares no conflicts of interest.

\section{References}

1 Kovesdy CP, Kalantar-Zadeh K. Review article: biomarkers of clinical outcomes in advanced chronic kidney disease. Nephrology (Carlton). 2009 Jun;14(4):408-15.

2 Ortiz A, Massy ZA, Fliser D, Lindholm B, Wiecek A, Martínez-Castelao A, et al. Clinical usefulness of novel prognostic biomarkers in patients on hemodialysis. Nat Rev Nephrol. 2011 Nov;8(3):141-50.

3 Pacher P, Kunos G. Modulating the endocannabinoid system in human health and disease-successes and failures. FEBS J. 2013 May;280(9):1918-43.
4 Gaoni Y, Mechoulam R. Isolation, structure and partial synthesis of an active constituent of Hashish. J Am Chem Soc. 1964;86(8): 1646-7.

5 Pertwee RG. Targeting the endocannabinoid system with cannabinoid receptor agonists: pharmacological strategies and therapeutic possibilities. Philos Trans R Soc Lond B Biol Sci. 2012 Dec;367(1607):335363.

6 Gallelli CA, Calcagnini S, Romano A, Koczwara JB, de Ceglia M, Dante D, et al. Modulation of the Oxidative Stress and Lipid Per- oxidation by Endocannabinoids and Their Lipid Analogues. Antioxidants. 2018 Jul; 7(7):E93.

7 Moradi H, Park C, Streja E, Argueta DA, DiPatrizio NV, You AS, et al. Circulating Endocannabinoids and Mortality in Hemodialysis Patients. Am J Nephrol. DOI: 10.1159/000505444.

8 Moradi H, Park C, Igarashi M, Streja E, Argueta DA, Soohoo M, et al. Serum Endocannabinoid Levels in Patients With End-Stage Renal Disease. J Endocr Soc. 2019 Aug;3(10):1869_ 80. 